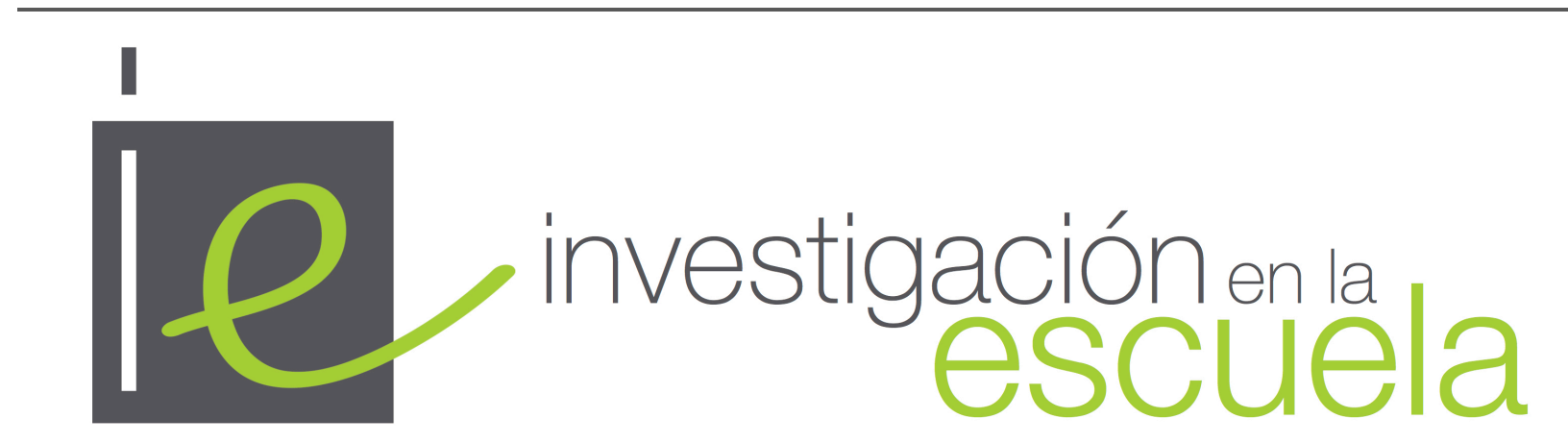

Revista internacional de investigación e innovación educativa

\title{
Currículum integrado: estrategias para la práctica
}

\author{
Francisco José Pozuelos Estrada y Francisco Javier García Prieto \\ Universidad de Huelva
}

España

Citación: Pozuelos Estrada, F. J. y García Prieto, F. J. (2020). Currículum integrado: estrategias para la práctica. Investigación en la Escuela, 100, 37-54. DOI: https://dx.doi.org/10.12795/IE.2020.1100.04

Resumen: El presente artículo expone el currículum integrado como un referente bien asentado en la tradición innovadora desde los inicios del siglo XX, alternativa que -aunque minoritaria frente al modelo convencional disciplinar- se puede encontrar en múltiples tentativas de cambio, reforma e innovación de momentos muy diversos de nuestra reciente historia educativa. Su presencia en las denominadas "pedagogías emergentes" corrobora su dinamismo y actualidad. El nivel de relación que se establezca entre las materias, así como el amplio abanico de estrategias descrito en este trabajo, expresan como los distintos asuntos, conocimientos y propósitos pueden acomodarse en un modelo curricular que se define por su flexibilidad, intención funcional y participación. Con un sentido ilustrativo, se describen y explican diferentes modelos y secuencias prácticas pero que en esencia comparten un bagaje pedagógico que les caracteriza y diferencia de otras propuestas más preocupadas por la transmisión mecánica y academicista del conocimiento escolar.

Palabras clave: "Currículum"; "innovación educativa"; "estrategias"; "trabajo colaborativo"; "evaluación formativa"; "modelo curricular".

\section{Integrated curriculum: strategies for practice}

Abstract: This article presents the integrated curriculum as a reference point implemented in the innovative tradition since the beginning of the 20th century, an alternative that -although a minority 
compared to the conventional disciplinary model- can be found in multiple attempts at educational change, reform and innovation at very different times in our recent educational history. Its presence in the so-called "emerging pedagogies" corroborates its dynamism and actuality. The level of relation established between subjects as well as the wide range of strategies described in this work express how different subjects, knowledge, and purposes can be accommodated in a curricular model that is defined by its flexibility, functional intention, and participation. In an illustrative sense, different models and practical sequences are described and explained, but which in essence share a pedagogical background that characterizes them and differentiates them from other proposals more concerned with the mechanical and academic transmission of school knowledge.

Key words: "Curriculum"; "educational innovation"; "strategies"; "collaborative work"; "formative evaluation"; "curricular model".

\section{Programme d'études intégré: stratégies pour la pratique}

Resumè: Cet article présente le curriculum intégré comme une référence bien établie dans l'innovation depuis le début de la s. XX. Sa présence dans les «pédagogies émergentes» d'aujourd'hui corrobore son dynamisme et son actualité. Le niveau de relation établi entre les matières ainsi que les stratégies décrites tout au long de ce travail expriment comment les différentes connaissances et finalités peuvent être intégrées dans un modèle curriculaire qui se définit par sa flexibilité, son intention fonctionnelle et sa participation. À titre d'illustration, différents modèles et séquences pratiques sont décrits et expliqués, mais ils partagent une base pédagogique qui les différencie des autres tentatives plus concernées par la transmission académique des connaissances scolaires.

Mots-clés: "Programme d'études"; "innovation pédagogique"; "strategies"; "travail en collaboration"; "évaluation formative"; "modèle de programme d'études".

\section{Introducción}

El planteamiento curricular integrado no es, contrario a lo que muchas veces pueda pensarse ${ }^{1}$, una novedad, cuenta con una larga y fructífera tradición (Hargreaves, Earl, Moore y Manning, 2001; Franklin y Johnson, 2006). Ya en los primeros movimientos preocupados por transformar la enseñanza en el marco de la Escuela Nueva aparece la globalización (integración, interdisciplinariedad, concentración de contenidos, etc.) como uno de los principios más sólidos y repetido (Kilpatrick, 1918; Freinet, 1927; Dewey, 1918; Decroly, 1932; etc.).

No obstante, y como efecto de su experimentación este principio ha ido evolucionado y adoptando matices diversos (Pozuelos, 2007). Aun así, es cierto que en este recorrido podemos encontrar periodos en los que su implantación adquiere una desigual atención: frente a momentos de amplia aceptación podemos hallar otros en los que se reduce su reconocimiento y práctica (Marsh \& Willis, 2007). Y ello viene influido, como apuntan Frankling y Johnson (2006) o Beane (2005), por las tensiones que en diferentes periodos se han ido sucediendo entre perspectivas enfrentadas que entienden, por un lado, el currículum como una propuesta con intención funcional, de acuerdo con las necesidades de los estudiantes y en base a la experiencia (progresista) o, desde otra postura (conservadora), la que se inclina por el desarrollo de las asignaturas según un minucioso orden

\footnotetext{
${ }_{1}$ Actualmente es frecuente leer artículos en los periódicos generalistas que aluden a la novedad que implica el conectar las materias en torno a una determinada temática, sirva de ejemplo el reportaje "Nuevos tiempos, nuevos profes" publicado por el País Semanal y firmado por Elena Sevillano, domingo 8 de septiembre de 2013 https://elpais.com/elpais/2013/09/11/eps/1378930400 452755.html; el de Natalia López Pevida (2018) https://elpais.com/elpais/2018/09/06/buenavida/1536234101 617950.html o el de M. Tomás Muñoz (2019) https://www.elconfidencial.com/sociedad/2019-04-08/santander-futuro-personas-leccion-seis-profesoresbra $1867322 /$
} 
académico, dentro de la estructura de las disciplinas con objeto de asegurar la adquisición de un conocimiento estrictamente intelectual y gestionado en función de unos objetivos de rendimiento. Como exponen Frankling y Johnson (2006), esa rivalidad entre las propuestas educativas centradas en los estudiantes y las centradas en las disciplinas no es algo exclusivo del escenario nacional americano, se ha producido, en prácticamente en todos los países.

Esa trayectoria nos muestra que la integración curricular constituye una referencia viva, dinámica y con potencial demostrado a la hora de organizar y abordar los conocimientos que se desarrollan en la enseñanza escolar (Dowden, 2007). Es más, los movimientos de cambio e innovación surgidos desde las aulas actuales lo han recuperado y actualizado hasta convertirlo en uno de sus pilares más significativo (Pozuelos, Romero, García y Morcillo, 2010; Martínez Celorio, 2016; Trujillo, 2019; Wert, 2019). Y, unido a esto, también contemplamos -aunque eso sí, con desigual complejidad y nivel de calado- su reiterada alusión en el marco de la reforma curricular de determinados territorios como Finlandia (García Ajofrín, 2015); Singapur (Lam, Alviar-Martin, Adler \& Sim, 2013), Quebec (Araujo-Oliveira, Lenoir, Morales-Gomes y McConnell, 2011), etc. En esta misma perspectiva, cualquier tentativa dirigida a promover una enseñanza alternativa orientada a un conocimiento más funcional, cercano y relevante señala a la globalización como uno de sus descriptores más importantes (Carbonell, 2014). De alguna forma, y por motivos no siempre coincidentes, constituye en estos momentos, una tendencia en alza (Hoachlander, 1999). Para unos, porque la realidad laboral actual necesita de nuevos conocimientos y habilidades que se escapan al marco disciplinar y, en consecuencia, el entorno educativo debe enseñar según esas demandas productivas (Barron \& Darling-Hammond, 2008) y para otros, porque el currículum integrado promueve la humanización del conocimiento, favorece el compromiso del alumnado con su realidad, incitándole a la participación responsable a la vez que permite abordar los problemas sociales y culturales que realmente afectan a la sociedad de hoy, en definitiva, porque la escuela tiene entre sus cometidos más relevantes la formación de una ciudadanía comprometida con la cultura y sociedad democráticas (Guarro, 2001). Como comprobamos se alude a postulados bien diferentes e implican posiciones radicalmente distintas, pero ambas dirigen la mirada a la necesaria conexión sustantiva entre los conocimientos. Por todo ello, para algunos se ha constituido casi en una moda, basta para esto echar una mirada a los periódicos generalistas cuando deparan en la enseñanza actual.

Como marco general de referencia, podemos decir que la integración curricular responde, básicamente, a la posibilidad de organizar y presentar el currículum educativo a partir de un eje temático que relaciona los distintos contenidos de forma significativa y de modo que resulte más reconocible y comprensible para los escolares en la medida que responde a su realidad y a las situaciones concretas que son capaces de atraer su atención e interés lo que les permite una comprensión más profunda y auténtica (Savage, 2003; Beane, 2005; Boss, 2011). Frente a la convencional y asentada fragmentación y distribución en asignaturas separadas con escasa representatividad fuera del marco académico -perspectiva esencialista- (Posner, 2004), el currículum integrado incorpora la idea de unidad entre las distintas formas de conocimiento y las disciplinas perspectiva progresiva y reconstruccionista- (Posner, 2004). Pero esto admite, expresiones prácticas y teóricas bien diferentes.

Como decíamos, el denominado currículum integrado alude a un concepto que admite muchos matices y diferentes niveles de complejidad a la hora de establecer las distintas relaciones entre los conocimientos. Desde esa panorámica hallamos iniciativas que yuxtaponen los contenidos del currículum en torno a un tópico concreto (concentración o pluridisciplinariedad), otras que pretenden la combinación y complementariedad entre materias (interdisciplinariedad) y aquellas que trascienden a las asignaturas y se funden con las más diversas formas de organizar y considerar el conocimiento escolar, es el denominado enfoque transdisciplinar (fusión). Ese continuo que acabamos de perfilar abre un abanico inmenso de posibilidades y escalas (Torres, 1994; Schumacher, 
1995; Fogarty, 2009). Pero estos grados no son estancos entre ellos, es más, en muchos casos es difícil establecer con precisión hasta dónde alcanza uno y en qué lugar comienza el siguiente y en buena medida ningún enfoque puede entenderse de forma definitiva y nítida, responde más a una perspectiva llena de gamas capaz de adaptarse a las más diferentes intenciones que cada experiencia quiera desarrollar (Drake \& Burns, 2004).

De cualquier modo, optar por una propuesta integrada del currículum conlleva necesariamente una selección y adecuación del conocimiento escolar. Los programas educativos de carácter convencional bien asentados en la tradición escolar suelen mantener un marcado sesgo enciclopedista y fragmentado en donde los contenidos tienden a acumularse de manera desmedida ${ }^{2}$ y, además, en la mayoría de los casos referidos de forma incomprensible para la mayoría de los alumnos lo cual origina que se olviden con gran velocidad, máxime si a esto le unimos el escaso valor práctico que tienen para la vida diaria de los estudiantes (Pérez Gómez, 2012).

El enfoque integrador más preocupado por promover la comprensión y reconstrucción de los significados que por la acumulación acrítica y reproductora de un cuerpo de conocimiento impuesto y cerrado, tiende a centrarse en mayor medida en la relevancia de los conocimientos que en la insustancial extensión de contenidos disciplinares que termina por impedir el tratamiento de cualquier tema con un mínimo de complejidad y profundidad (Sánchez Caballero, 2017; Coll, 2006; García Rubio y Ros, 2018)

Y obviamente se impone la necesidad de priorizar y seleccionar sólo aquellos saberes que verdaderamente tienen sentido con el proyecto que se quiere desarrollar aligerándose con ello, el currículum escolar, de contenidos secundarios que en la mayoría de los casos son únicamente un relleno de escaso valor educativo. Y, por otro lado, se enriquece con aportaciones derivadas de las nuevas formas del saber y proceder -inteligencias múltiples, competencias, "habilidades blandas"(Howell, 2003; Barron y Darling-Hammond, 2008).Y es que desarrollar una experiencia integrada impulsa un proceso que pone en juego formas de saber y contenidos que pasan desapercibidos en el marco de las asignaturas y, por ello, pierden su potencial formativo al estar situados en un espacio no identificado por ninguna materia concreta: trabajo en equipo, comunicación efectiva, creatividad, pensamiento crítico, empatía, etc. (Meneses, 2020; Buxarrais, 2013).

Dinámica que explica el proceso de enseñanza y aprendizaje según una actividad dialogada entre todos los participantes y capaz de considerar el conocimiento como reconstrucción colaborativa a partir de asuntos relevantes que se sustancian en algo más que las asignaturas académicas (García-Varcálcel y Basilotta, 2017; Fraser, 2013).

\section{El currículum integrado en el aula: algunas estrategias afines}

A la hora de integrar los contenidos y materias en una secuencia de aprendizaje se han propuesto diferentes vías y modalidades. Entre los enfoques o procesos más extendidos se encuentran los que giran alrededor de un tema o tópico, los que se centran en la resolución de un problema o situación inquietante, los que desarrollan algún tipo de investigación o indagación escolar y los que pretenden abordar algún proyecto o materialización práctica de una idea. Todos comparten las bases e ideas ejes pero cada uno, a su vez, sigue su propia estructura y lógica de desarrollo ya que independientemente de la naturaleza del esquema o de su origen, cada proyecto tendrá un tema o problema unificado que orientará al docente y alumnado y ayudará a adquirir comprensiones y conocimientos de más alto nivel de complejidad cognitiva -desarrollo de habilidades superiores-. Veamos de forma sucinta algunas de estas diferentes vías y modalidades.

\footnotetext{
${ }^{2}$ En el Blog de Feito (http://rfeito.blogspot.com.es/) se puede leer una síntesis del consenso alcanzado sobre el sobrecargado currículum oficial en las distintas esferas de los representantes de la comunidad educativa.
} 


\section{Unidades didácticas integradas}

En algunos casos se expone la posibilidad de partir de un "tópico" o asunto que no es tratado por ninguna materia en concreto y que, sin embargo, afecta a todas, o a varias, de alguna manera, por ejemplo: la contaminación. Con esta referencia se combinan las disciplinas afectadas a partir del "tema" que le da nombre, lo que permite articular el currículum desde una perspectiva integrada. Existen, sin embargo, distintas modalidades de llevarlo a cabo en la práctica, desde una simple reestructuración del "programa" con el fin de que todas las materias, en su parcela, estudien algo relacionado con el asunto tratado (modelo aditivo); hasta romper la separación entre las diferentes disciplinas y relacionar el contenido de las diversas áreas y conocimientos en el marco de una experiencia integrada.

Estos "temas" también pueden variar según el nivel de "estabilidad" que se le conceda. Unos, por su relevancia permanecen como ideas-fuerzas que articulan y dan sentido al currículum de manera que tengan una continuidad y se aseguren de este modo el desarrollo equilibrado de los conocimientos que se consideran básicos para un ciclo o etapa (por ejemplo, "la alimentación"). Otros, por su carácter puntual, se centran únicamente en un aspecto concreto y coyuntural que atraen momentáneamente la atención del alumnado, suelen estar relacionados con acontecimientos muy precisos (por ejemplo, "las olimpiadas") por lo que no será necesario planificarlos siguiendo una progresión, ni relacionarlos con otros pues tienen un principio y fin en sí mismos.

Como plantea Fraser (2000) este formato se caracteriza por la intervención decisiva del docente que es quien realmente señala la temática, planifica la experiencia y, en todo momento, se busca cubrir el plan de estudio. El nivel de negociación con el alumnado queda bastante relegado (Lacueva, 2017), prácticamente, sólo se espera que lo desarrolle y alcance los fines previamente fijados.

Igualmente, la versión más esquemática de esta modalidad es la que siguen los manuales escolares que algunas editoriales han presentado como producto comercial destinado a dar respuesta a la cada vez más demandada actualización de la enseñanza y los textos escolares.

Por último, solo decir que en realidad -en la mayoría de los casos- encierra una reordenación de los contenidos (enfoque multidisciplinar) a partir de supuesto temas de interés, o lo que es igual: enseñar lo mismo de otra forma.

\section{Los proyectos de trabajo: investigar para aprender}

Los proyectos de trabajo basados en la investigación escolar (Pozuelos, 2007) se basan en el aprovechamiento de la curiosidad y el interés expresados por los escolares. Tienen su origen en las preguntas e interrogantes que se hace el alumno en relación con diferentes fenómenos, acontecimientos o hechos, esto es, que el conocimiento escolar se presente como una respuesta destinada a una cuestión previamente formulada.

Con esta referencia se organizaría el proceso de enseñanza/aprendizaje, para ello se tratan diferentes contenidos pero no en orden a unas materias sino con el fin de lograr unas producciones fundamentadas a través de unos procesos valiosos desde un punto de vista educativo. Estas cuestiones a veces son planteadas directamente por el alumnado pero, por regla general, suelen estar motivadas por alguna experiencia o encuentro que motivan discusiones y dudas que precisan un cierto nivel de estructuración e información, aquí el grupo del aula (alumnado y profesorado, cada uno en su grado de responsabilidad) desempeñan un papel fundamental en el proceso. Y lo verdaderamente importante es que los participantes hagan suyo el proyecto, que emerja como una motivación interior impulsada por el deseo de saber y hacer.

Es común que al diálogo inicial le siga una serie, más o menos ordenada, de interrogantes parciales o subpreguntas que permiten desarrollar aspectos concretos de la investigación así cada 
grupo puede abordar un apartado y posteriormente lo organizará con el fin de comunicarlo al resto de participantes.

Una de las características que mejor define a un proyecto de trabajo (Pecore, 2015) consiste en la implicación directa de los estudiantes en todo el proceso. El objeto de estudio y la secuencia de experiencias, tareas y producciones es más impulsado que dirigido.

Pero esto no es una invitación a seguir una secuencia errática, improvisada o caprichosa, pues como exponen Markham, Larmer \& Ravitz (2003), un proyecto se apoya en un proceso sistemático articulado en torno a un asunto relevante que responde al interés de todos los participantes y del que se desprenden preguntas motivadoras. Las respuestas se originan como efecto de la colaboración en base a múltiples consultas donde la creatividad y las destrezas de orden superior (Torres, 2014) encuentran su mejor expresión.

$\mathrm{Y}$ algo importante, insistimos, desarrollar el currículum a partir de proyectos implica una planificación flexible y compartida, que orbita en torno a experiencias sustantivas capaces de producir aprendizajes relevantes más que en los resultados previamente señalados. Se trata antes de una "hipótesis de trabajo" que de una planificación precisa cerrada con antelación.

A veces estos proyectos e investigaciones sufren procesos desmotivadores para el alumnado (Engel, 2011) bien por lo efímero del interés bien por el calado de los contenidos, la dificultad o superficialidad de las actividades y experiencias, la ausencia de recursos y materiales apropiados, etc. Es, por todo ello, que el profesorado en este tipo de estrategia de aula juega un papel especialmente relevante y delicado: regular el proceso adecuándolo suficientemente a cada caso, pero sin eliminar el protagonismo que el alumnado debe tener a lo largo del mismo.

Por último, en este sintético apunte sobre el trabajo por proyectos, quisiéramos destacar que a lo largo de un proyecto se establecen conexiones entre conocimiento y formas de aprender imposible de lograr siguiendo el orden convencional de los contenidos disciplinares lo cual repercute positivamente en su formación y la comprensión de lo que aprende. Y así ha sido expuesto por las investigaciones que se han desarrollado para conocer el alcance y mejoras que se obtiene cuando un docente decide implementar el trabajo por proyecto en sus clases (Bredley-Levin, Berghoff, Seybold, Sever \& Blackwell, 2010; García-Varcálcel y Bisolotta, 2017).

Los proyectos por su naturaleza temática y abierta a la investigación encajan bien con las transdiciplinariedad, pues se centran en las relaciones complejas que se derivan de confeccionar respuestas y generar producciones en base a preguntas y necesidades más que en dar cuenta exclusivamente de los contenidos académicos dispuestos en las asignaturas.

\section{El aprendizaje basado en problemas: dilemas que invitan a investigar}

En ocasiones se propone partir de problemas que, de alguna manera, afectan al alumnado o que, al menos, atraen su atención. Según este modelo los diferentes conocimientos se aglutinan en función de alguna situación cotidiana o prevista que produce, por un lado, un cierto grado de incertidumbre y, por otro, provoca la movilización de variados recursos intelectuales y materiales al objeto de encontrar una solución fundamentada. Implica el razonamiento como eje para dar la respuesta frente a la simple aplicación de un recurso o una fórmula exacta y mecánica. McGrath (2002) definió el aprendizaje basado en problemas como un método de aprendizaje en grupo que usa dilemas reales (la descripción de un caso puede ser un buen recurso para ello) como estímulo para desarrollar habilidades de solución de problemas y adquirir conocimiento específico.

Se pretende sobre todo fomentar la capacidad lógica, de diálogo, de intervención, de toma de decisiones fundamentadas e incluso de apuntar soluciones que de alguna manera les comprometa e involucre personal y colectivamente y no, en consecuencia, de la repetición y memorización de respuestas terminadas o conductas preestablecidas. Esta estrategia, como apunta Pozo (2016), configura un procedimiento abierto que admite diversos caminos para llegar a la solución. Para ello 
es preciso establecer un proceso que incluye la deliberación sobre la concreción del problema, planificación con el fin de expresar soluciones originales y sólidas, presentación de resultados y discusión sobre la validez que encierra. Estrategia que se aparta, de esta forma, de la aplicación de simples rutinas o técnicas transmitidas previamente por el profesorado.

Para su empleo en el aula se aconseja trabajar en pequeños grupos que colaboren para abordar un problema e identifiquen lo que necesitan para dar respuesta y alcanzar soluciones basadas en evidencias y razonamiento. En cualquier caso, el alumnado es el verdadero protagonista del proceso que, en compañía del docente, trabaja de forma activa en la producción de resultados contrastados. El profesorado apoya, invita a recapacitar, manifiesta dudas, facilita recursos, en definitiva, fomenta la autonomía y la necesidad de argumentar en base a pruebas previamente sopesadas y verosímiles, es decir, como apunta Delval (2010), ponen las condiciones para que los estudiantes aprendan mediante su propia actividad. Reflexión compartida sobre el problema inicial, planificación y tratamiento de la información, aplicación de la respuesta en situaciones diversas, deliberación cooperativa sobre los resultados y síntesis, describen la secuencia que recorre una experiencia sustentada en el aprendizaje basado en problemas.

Cuando el alumnado se enfrenta ante los desafíos estimulantes de un problema recurre a distintas formas de conocimiento y contenidos pues raramente un asunto así formulado se circunscribe a una materia aislada. La integración y conexión entre aprendizajes emerge de forma natural.

Es fundamental para esta estrategia conectar los problemas que se trabajan en la escuela con la vida de los estudiantes a los que se les pide que los acometan pues, como se ha demostrado en reiteradas ocasiones (Silver, Smith y Nelson, 1997), el tratamiento de problemas claramente academicistas (como pueden ser los problemas cuantitativos) termina por resultar algo poco funcional, al ser abordados por el alumnado como una tarea escolar que difícilmente pueden trasladar a un contexto distinto del que se aprendió. Junto a esta limitación también se le atribuyen otras como son la exigua significatividad, la tendencia a la rutinización y, por tanto, el escaso interés que finalmente provoca en el estudiantado. Todo ello incitado por esa tendencia instrumental y cerrada que impulsa más que a tratar con problemas, a "reconocerlos" e identificarlos con modelos tipo para los que ya se tienen respuestas y procedimientos disponibles y automáticos: "Gran parte del estudiantado no es capaz de abordar problemas nuevos, y cuando dan una respuesta correcta los procedimientos utilizados por ser mecánicos no les permite entender nada" (Mora y García, 1998, p. 16).

En definitiva, el aprendizaje basado en problemas obedece al fracaso demostrado de la simple memorización o aplicación automática de fórmulas universales cuando se pretende transferir a relacionar conocimientos en situaciones diversas y cambiantes.

$\mathrm{El}$ aprendizaje basado en problemas ha demostrado una sólida validez, sobre todo, cuando se pretende reforzar la deducción, la argumentación, la formulación de hipótesis concretas, la explicación lógica y la comprensión de la ciencia (Barron y Darling-Hammond, 2008)

El aprendizaje basado en problemas como expresan Barron y Darling-Hammond (2008) resulta "un primo cercano del aprendizaje basado en proyectos" (p. 4), la diferencia quizás reside en que el primero busca respuestas a dilemas complejos en base al diálogo argumentado y los proyectos persiguen la elaboración de productos que desarrollen las habilidades y conocimientos que se desprenden del tratamiento de las preguntas sugeridas (García Varcálcel y Basilotta, 2017).

\section{Design Thinking: crear para aprender}

Diseñar y hacer algún tipo de objeto e incluso la elaboración de un montaje puede ser un buen referente para integrar los diversos conocimientos escolares. Desde esta perspectiva no basta con informarse o tratar ciertos conocimientos, es preciso hacerlo y enterarse haciéndolo, es el 
resultado de combinar: interés, experiencia y acción; todo ello relacionado con habilidades como empatía, colaboración, curiosidad y creatividad. Estamos hablando de una organización del proceso de enseñanza/aprendizaje que pretende conjugar teoría y práctica a la hora de abordar los contenidos, lo cual supera a ciertas tendencias reduccionistas que vinculan a esta estrategia con conocimientos exclusivamente manipulativos. De hecho, cuando leemos sobre el "Design Thinking" (Brown, 2010) una idea que se repite está relacionada tanto con la creatividad como con la necesidad de vincular la reflexión con la producción efectuada.

Este modelo que tiene su origen remoto en la industria propone, por tanto, plasmar el resultado de una experiencia educativa en algún tipo de producción física y para ello habrá que buscar información, ordenar y calcular datos, conseguir recursos adecuados, plantear modelos, trabajar solidariamente y en colaboración, es decir, una mezcla hábil entre pensamiento y acción que puede sintetizarse en el empleo sistemático de elementos visuales (gráficos, maquetas, prototipos, infografías, vídeos, fotos, diagramas, etc.) que ayuden a trabajar y comprender determinados conceptos complejos de forma más intuitiva y atractiva pues se basan en el poder y seducción de las imágenes y las "historias" para mostrar y hacer comprender realidades y problemáticas cada vez más complejas (Vinyets, s/f).

En su puesta en práctica encontramos dos vías. Una, en la que el docente expone un ejemplo, así como su proceso de producción y tras esto propone nuevos formatos a partir de la iniciativa de los escolares. Y otro, en el que el docente plantea un reto que los estudiantes abordan según su propio ingenio, en este caso los apoyos por parte del profesorado se administran cuando son demandados o como efecto de las reiteradas revisiones compartidas que durante las distintas fases se generan. El rol docente se aparta del transmisor de respuestas directas para adoptar la imagen del promotor que apoya durante la experiencia y facilita un ambiente óptimo para la creación y el aprendizaje compartido. Lo importante radica en pensar y hacer. Y, en definitiva, no perder la creatividad con la que los escolares acceden a la escuela y que desgraciadamente desaparece según avanza en el sistema educativo (Steinbeck, 2011).

El desarrollo de un proyecto proporciona saber y experiencia conjuntamente. Profundizando un poco más, resulta una estrategia bastante flexible pues permite aplicar criterios muy amplios a la hora de organizar la experiencia. Sirvan de ejemplo algunas de las orientaciones que seguidamente se exponen.

Grupos muy distintos. Es posible realizar actividades en las que los equipos de trabajo son de procedencia muy dispares (de la misma aula, del ciclo e, incluso, interciclos) lo que enriquece las relaciones y dimensiona el aprendizaje como una experiencia a compartir dentro de la diversidad. El equipo y las distintas miradas configuran un tejido sólido con mayor base creativa que la perspectiva personal y aislada.

Temáticas variadas. Son múltiples los objetos, montajes o tareas a realizar, podemos encontrar ejemplos que tratan desde un periódico al cuidado de un huerto o la confección de un prototipo, entre otros.

Materiales versátiles. La realización de un proyecto precisa, por la variedad de actividades que se ponen en juego, de una gama muy extensa de medios y recursos: manipulativos, impresos, audiovisuales, contacto directo, digitales, etc., lo cierto es que para implementar las actividades previstas es necesario salirse del uso tradicional de los materiales escolares. Y, como decíamos, destacan todos aquellos medios que promueven la construcción visual de las aportaciones.

Conocimientos distintos y complementarios. Un proyecto basado en "design thinking" incluye distintas habilidades y competencias en torno a una producción significativa. Va más allá de las consabidas asignaturas adentrándose de forma natural en las distintas inteligencias y habilidades superiores que en poca proporción encontramos en la denominada enseñanza convencional 
(magistral). Coincide con el modo de trabajar en muchas situaciones prácticas actuales: revisar, concretar, analizar, crear y evaluar.

Escenario dinámico de aprendizaje. El contexto del aula adopta una imagen mucho más funcional, flexible y adaptada a la participación y la comunicación. En este sentido, se aparta tanto del frío escenario neutro como de la decoración escolar, se busca sobre todo la humanización donde la comunidad que aprende dispone su espacio como un lugar para la creación y muestra de su trabajo. Flexibilidad horaria y de agrupamientos, accesibilidad de los recursos y materiales, exposición de resultados visibles, ubicación funcional del mobiliario, etc., generan una imagen del aula bien diferenciada del pasivo y frío modelo tradicional.

Y aunque se han definido fases y secuencias lógicas (ver por ejemplo https://dschoolold.stanford.edu) lo cierto es que, como apunta Marta Lago (s/f), el sentido profundo del Design Thinking radica en una forma innovadora de acometer el aprendizaje donde el protagonismo compartido de todos los participantes canaliza la creatividad para la relevancia del saber. Implica, por tanto, un cambio de mentalidad más que una técnica o método de enseñanza.

Dicho lo cual comprobamos lo valioso que puede resultar su aplicación en un centro pues contribuye en buena medida a redimensionar el currículum desde una perspectiva innovadora dentro de un clima de colaboración, intercambio, actividad y creatividad en su sentido más amplio.

En la actualidad, el "design thinking" conecta con la cultura "maker" y, desde esta mirada, su proximidad a las STEM -Ciencia, Tecnología, Ingeniería y Matemáticas- (Meneses, 2020) le encuadran bien con un perfil interdisciplinar pero apto para todas las edades. Y como expone Zurdo (2018), aunque realmente coincide con un proyecto de base tecnológica esto no le impide abordar los más distintos contenidos y habilidades de forma relacionada. Aquí el objeto de estudio constituye el referente más diferenciador. Aun así, una vez más, vemos como los límites son más precisos en la declaración teórica que en su dimensión práctica.

\section{Aprendizaje servicio: el currículum como motor social}

Apoyarse en el análisis e indagación de las necesidades que afectan a la comunidad para actuar en su beneficio ha sido una de las situaciones a la que más se ha recurrido para desarrollar estrategias globalizadoras.

Se parte de la importancia de establecer relaciones de interacción entre el niño y su propio entorno comunitario con objeto de que conozca, de forma fundamentada y experiencial, la realidad en la que vive y que, como le afecta de múltiples formas, deberá aprender a analizar para intervenir críticamente. Significa un cambio educativo radical en la medida que los participantes y el contexto social en el que viven protagonizan un proceso en el que se aplican las competencias y contenidos curriculares en situaciones reales con el objetivo de lograr un beneficio social (Zorrilla, Capella y Gil, 2016).

Este proceso de investigación e intervención sobre el entorno no sólo reporta un conocimiento más o menos cercano y contextual, también conlleva la acción sobre la misma realidad y para ello no basta el simple estudio natural o social sino un enfoque más amplio que contribuya a establecer relaciones complejas y propuestas de participación fruto de diversas fuentes de conocimiento y experiencia (escolar, social y alternativo), pues la adquisición de un saber fragmentado y parcelado resulta inútil para afrontar la complejidad de los problemas sociales de una determinada comunidad. Como afirma Cieza (2010) se enfatiza tanto el aprendizaje académico que se desarrolla en el aula como la realización de un servicio a favor de los problemas y necesidades reales detectados en la comunidad de forma que ambas dimensiones se complementan.

Como podemos ver, esta propuesta basa su estrategia en tres aspectos complementarios: el análisis crítico del medio (aprender a interrogar); la búsqueda, selección y relación de datos e información (aprender a argumentar) y el planteamiento de soluciones que signifiquen un 
compromiso activo (aprender a comprometerse). Es decir, el fomento de una ciudadanía responsable que basa su intervención y opiniones en argumentos sólidos y fundamentados, más allá de la simple intuición momentánea. Como afirma Batlle (2011) se pretende formar personas críticas y consecuentes con la realidad en la que viven a la vez que ofrecen un servicio a la comunidad.

Lo cual se traduce en un proyecto que cumple una doble función, por una parte se convierte en una estructura de análisis crítico de la realidad, basándose para ello en una amplia gama de conocimientos y experiencias, con el fin de lograr una interpretación global; y, por otro, representa un instrumento de acción cultural y transformadora desde la propia escuela. En este marco tanto Álvarez y Silió (2015) como Zorrilla, Capella y Gil (2016) entre otros y en directa relación con la Writing@CSU de la Universidad de Colorado, consideran que los elementos clave de un proyecto de aprendizaje servicio giran en torno a unos elementos comunes: 1. Adquisición de los contenidos dispuestos en el currículum escolar. 2. Los aprendizajes son fruto de la intervención relevante en el marco de la comunidad que recibe el servicio. 3. El alumnado juega un papel decidido e informado. 4. Implica un proceso organizado que asegura la viabilidad formativa de la experiencia.

Y todo ello dentro de una flexibilidad metodológica que le acerca a una forma bastante reconocible de desarrollar la práctica escolar para buena parte de los docentes, de ahí su expansión en diferentes lugares, países y etapas educativas. En este sentido existen colectivos como la Red Española de Aprendizaje Servicio (https://aprendizajeservicio.net), la Red Nacional de Aprendizaje Servicio (http://www.rease.cl) o "Design for Change" (http://www.dfcspain.com), que se inspiran en este planteamiento.

Parte de la expansión de este formato educativo se debe a que asegura la conexión de los distintos contextos en los que el alumnado experimenta su vida (familiar, escolar y social) y se les brinda la oportunidad de participar de forma responsable, solidaria y creativa de modo práctico y con sentido de utilidad personal y social.

En fin, contactos con el medio y la comunidad que les permitirán discurrir acerca de la realidad y su transformación pues de otra forma la escuela, entendida como una simple institución dedicada a la transmisión de datos y conocimientos descontextualizados, puede pasar a un segundo orden de importancia cultural ante la imposible competencia que tendría que mantener con la poderosa tecnología de la información.

Como expone Uruñuela (2018) el Aprendizaje Servicio mantiene similitudes con los planteamientos de Dewey y el "aprender haciendo" pero en este caso se trata de participar en un servicio a la comunidad con objeto de mejorarla. Esta mirada crítica y socialmente activa es realmente su seña de identidad. Además, como este mismo autor plantea, admite según el servicio prestado un enfoque disciplinar en el seno de una sola asignatura o interdisciplinar en la medida que varias materias se combinan con un propósito compartido. Pero su mejor espacio educativo lo encuentre, asegura Uruñuela, en la dimensión transdisciplinar que funde conocimientos y contenidos diferentes y complementarios con objeto de que el aprendizaje y el servicio lleguen a su máxima expresión formativa y transformadora.

\section{Aprendizaje en profundidad: educando desde la imaginación}

Últimamente, y aun realmente poco explorado, está planteándose una vía “narrativa” para elaborar propuestas globalizadoras de enseñanza. Consiste básicamente en apoyarse en la importancia que tiene, paradójicamente, la fantasía y la imaginación para la comprensión de la realidad en la mentalidad infantil pues, como asegura Egan (1994, p. 12), "la imaginación de los niños es la herramienta de aprendizaje más potente y enérgica" con la que cuentan.

El exceso de realismo y pragmatismo ha hecho que se olvide que el niño ve la realidad transfigurada por la fantasía y es esta la que insta a pensar en algo como posible, es la fuente de la invención, la creatividad y la generatividad (Egan y Judson, 2018). Es más, en la práctica, la mayoría 
de los maestros suelen utilizarla como referente educativo pues, su experiencia, les dice que estimula la inteligencia de diferentes formas, sirva de ejemplo la reflexión que hacen Silver, Smith y Nelson (1997) cuando expresan las ventajas que les aportaba relacionar las actividades de clase con distintas formas de narraciones, cuentos y poemas. En el mismo sentido se manifiestan interesados Barbosa, Alvesy Gonçalvez (1997) cuando explican cómo buscando un instrumento que les facilitara abordar la iniciación a la ciencia desde una perspectiva sólida, experiencial, interactiva y fundamentada, se percataron de que los cuentos infantiles, hábilmente tratados y adecuados, pueden tener estas cualidades, a la vez que conectan perfectamente con la dinámica infantil de relacionarse con las ideas abstractas. De alguna manera, se asegura, conecta con la forma natural de aprendizaje en la infancia.

Egan (1991, p. 36) propone que "las fantasías de los niños son formas primitivas y los primeros desarrollos de los conceptos más profundos y fundamentales que utilizamos para dar sentido al mundo y a la experiencia”. Para su desarrollo práctico se considera que partir de lo conocido significa apoyarse en los conceptos abstractos fundamentales que los niños desde muy temprano conocen (odio/amor; bondad/maldad; alegría/tristeza; miedo/seguridad...) y lo "desconocido" cualquier contenido que quisiéramos enseñar; así un cuento (una narración fantástica en general) y su desarrollo progresivo a partir de actividades y experiencias puede facilitar la selección y organización adecuada, para el alumnado, de conocimientos y valores y además constituye una finalidad inmediata capaz de sostener y dar sentido a todo el proceso de la unidad didáctica a través de un uso adecuado y coherente de imaginación e investigación, de problemas y misterios, de aventuras y conocimiento, que tanto significado tiene, como ya hemos dicho, para el mundo infantil (Lledó y Pozuelos, 1995). Es una forma de conectar emociones, imaginación e intelecto en el proceso de aprendizaje.

Tomando esta línea, el Imaginative Education Research Group -IERG- (https://ierg.ca) plantea el "aprendizaje en profundidad" como un proceso continuado destinado al tratamiento progresivo de un asunto a lo largo de toda la escolaridad con el fin de promover la comprensión compleja de una temática, convirtiendo el estudio escolar en un itinerario hacia su comprensión profunda lo cual le será útil para transferirlo a distintos escenarios tanto en términos de contenidos como de habilidades, competencias y emociones. Consiste, según sus autores (Grimaldo, Judson, Boullosa y Acuña, 2017) en una experiencia real que explora poco a poco las distintas herramientas cognitivas del ser humano desde lo fantástico, mítico, romántico filosófico e irónico. Lo imaginativo permite pensar no solo en lo que es posible sino también en lo que podría ser o es contado de otra forma, no vale para esta perspectiva únicamente el realismo racionalista imperante en la enseñanza formal empeñada en descarta cualquier atisbo de fantasía.

De esta forma planificar la secuencia de aprendizaje no se reduce a la organización de objetivos en sucesiones lógicas sino como una construcción sobre los poderosos conceptos abstractos que los alumnos poseen (bondad/maldad; avaricia/generosidad, etc.) y en torno a ello se selecciona un asunto relevante y atractivo en torno al cual se ajustan las distintas herramientas intelectuales y la secuencia de actividades que mejor se adapten para narrar esta historia, para concluirla es preciso buscar algún tipo de resolución al conflicto planteado por el "opuestos binarios" (Egan 1991; Egan y Judson, 2018).

El desarrollo curricular se alcanza de forma más sencilla, según sus autores, por el enlace que se provoca entre las emociones, su imaginación y el contenido de los diferentes contenidos y competencias. Pues el pensamiento infantil se estimula de forma más profunda cuando imaginación y emociones entran en juego. Y además se promueven destrezas que escapan a la lógica disciplinar de las asignaturas escolares. Representa un tránsito en profundidad en torno a una temática apasionante más que la consabida sucesión superficial de temas dispersos e irrelevantes.

El planteamiento longitudinal descrito por Boullosa (2017) a través del desarrollo de un tema tutorizado a lo largo de toda la escolaridad encierra una perspectiva claramente transdisciplinar e 
incluso en "espiral". No decimos que sea fácil en la estructura organizativa y curricular la escolaridad contemporánea pero sí abre nuestra mente a otros entendimientos más amplios y ambiciosos.

\section{Una breve conclusión}

Pero lo cierto es que son muy frecuentes las experiencias que se apoyan en aspectos variados de cada una de estas modalidades (ver figura 1) obteniéndose así procesos mixtos que se caracterizan por compartir un núcleo común cuyas líneas maestras radican en tener en cuenta los intereses del alumnado, diluir o redimensionar las tradicionales fronteras de los conocimientos y contenidos escolares, estructurar el tiempo académico de forma más flexible y dinámica, establecer una relación profesor-alumno más estrecha y colaborativa, enmarcar el escenario escolar de forma abierta para facilitar la comunicación y el acceso a la información.

En coherencia con lo anterior, el proceso gira alrededor de algunas cuestiones que involucran activamente al alumnado, la planificación profesor-alumno (dialogada) forma parte del desarrollo educativo, se establece una gran variedad de experiencias de aprendizaje y el conocimiento a tratar es mucho más complejo, pues se abarcan competencias y contenidos no incluidos tradicionalmente en el currículum escolar.

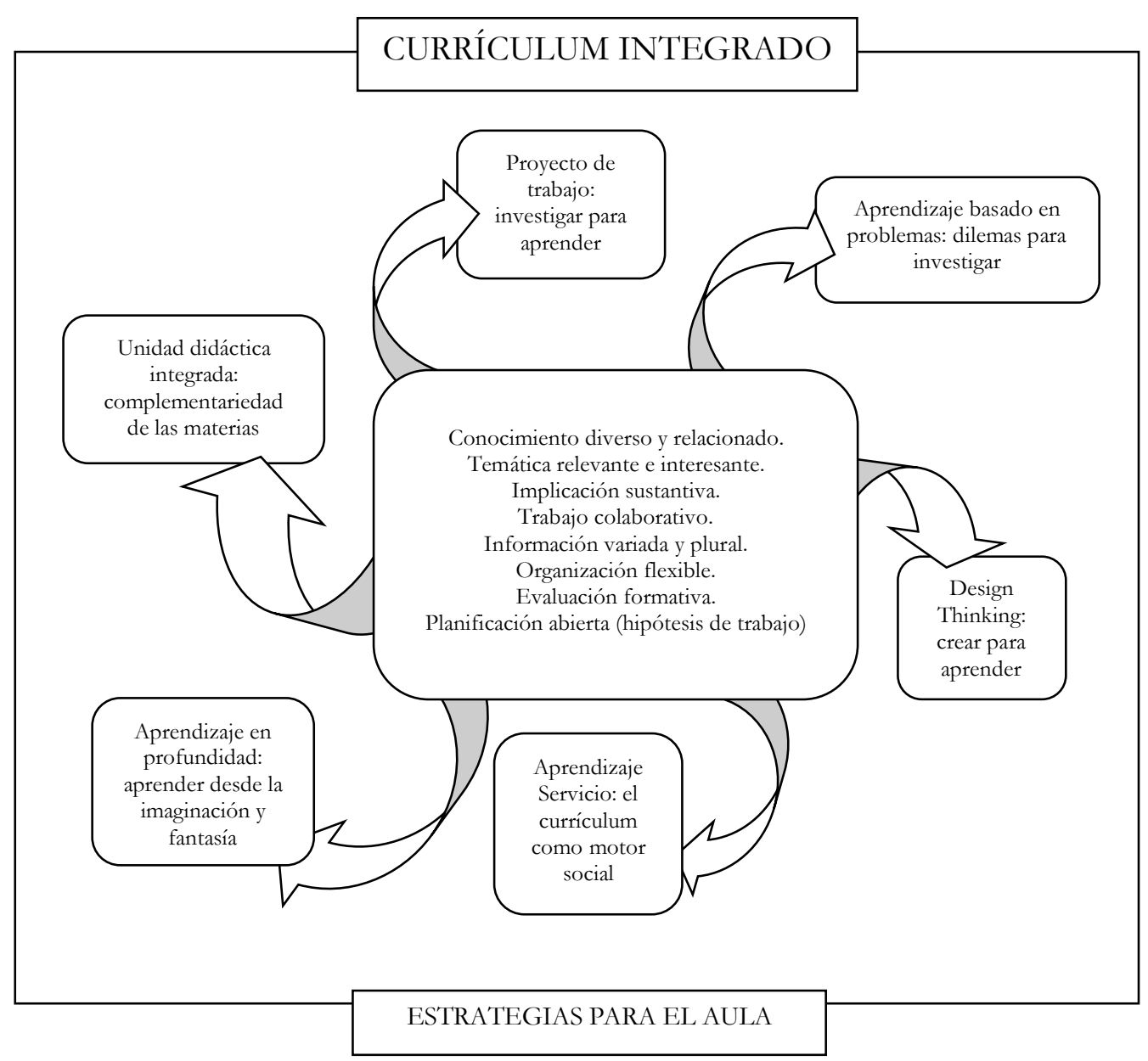

Figura 1. Currículum integrado, estrategias para el aula 
No obstante decidirse por un planteamiento curricular integrado no está exento de obstáculos. Muchos ya se han descrito (Beane; 2005; Fraser 2000; Hernández y Ventura, 1992; Pozuelos y Rodríguez, 2008; Drake y Burns, 2004) pero en este trabajo de forma sumaria, destacaríamos que encontrar y delimitar un tema que sea capaz de relacionar significativamente distintos tipos de conocimientos relevantes y que a su vez sea desafiante para los escolares, no es fácil. Es frecuente recurrir a asuntos estereotipados que no terminan de involucrar al alumnado o se basan en relaciones forzadas de contenidos académicos sin relación significativa más allá de formalismos supuestamente globalizadores. Lo que conduciría a lo que se ha denominado "metodología de la superficialidad" (Gil, 1987). Es decir, abordar en clase cualquier asunto de poca exigencia bajo el falso argumento del interés de los escolares.

Otro aspecto a considerar tiene que ver con la necesidad que el profesorado presenta a la hora de planificar su actividad docente. El desarrollo de un currículum integrado en la medida que aborda temáticas vivas que incluyen conocimientos diversos -y no sólo académicos- y promueven la implicación sustantiva de los escolares, escapa a la determinación estricta de la planificación (Carbonell, 2015) y muchos profesores llegan a sentirse intimidados cuando no tienen todas las actividades cuidadosamente preparadas con bastante tiempo de antelación.

Igualmente una buena parte de los docentes tiene la impresión de que con esta forma de presentar el currículum muchos contenidos importantes se diluyen o no son abordados con el rigor que estos merecen. Las pruebas externas y sus estándares, así como la implantación generalizada de los libros de texto en su formato más convencional refuerzan esos temores con más o menos firmeza según los casos.

Además, una experiencia integrada significativa y que estimule al alumnado y que les ayude a manejar y asimilar los contenidos propuestos exige el empleo de recursos que no siempre están al alcance del profesorado. Y esto, tanto para su elaboración como para su adquisición pues, aunque actualmente encontremos algunos proyectos (Cañal, Pozuelos y Travé, 2005), la mayoría de los materiales coinciden en poco con un modelo integrado del currículum.

Por último, observamos la presión institucional que valora sobre todo la transmisión de contenidos disciplinares a la vez que recela de cualquier cambio que perturbe la competencia profesional aprendida desde muy temprano y ampliamente asumida entre el colectivo docente (Monereo, 2009).

Aun así, y coincidiendo con diferentes autores e investigaciones, nos inclinamos a pensar que resultaría verdaderamente interesante tratar el currículum escolar a partir de la combinación de algunas de las estrategias apuntadas según el propósito de cada experiencia. Teniendo esto en cuenta podemos decir que la riqueza, diversidad y posibilidad de adaptación es tan amplia como distintas son las necesidades y exigencias de los diferentes contextos y realidades educativas.

\section{Agradecimientos}

Financiado por el proyecto I+D+i en el marco del programa operativo FEDER Andalucía 20142020 "Anatomía del cambio educativo: las escuelas ante el reto de la innovación pedagógica" (UHU1256182).

\section{Referencias}

Álvarez, C. y Silió, G. (2015). El aprendizaje servicio y las comunidades de aprendizaje: dos proyectos escolares innovadores que se enriquecen mutuamente. Enseñanza \& Teaching, 33, 43-58. 
Araujo-Oliveira, A., Lenoir, Y., Morales Gomes, M. A. y McConnell, A. C. (2011). Práticas interdisciplinares no ensino primário: concepçoes de profesores e futuros profesores no Québec. Educação e sociedade, 32(117), 1125-1145.

Barbosa, M. C.; Alves, L. y Gonçalvez, R. A. (1997). Una propuesta: enseñar física a niños de grados elementales. Enseñanza de las Ciencias, 15(2), 273-277.

Barron, B. \& Darling-Hammond, L. (2008). Teaching for meaningful learning. San Francisco: The George Lucas Educational Foundation. Disponible en: https://edut.to/2Hgs'TnR

Batlle, R. (2011). ¿De qué hablamos cuando hablamos de aprendizaje-servicio? Crítica, 972, 49-54.

Beane, J. A. (2005). La integración del currículum. Madrid: Morata.

Boss. S. (2011). Integrated studies: a short history. Edutopia: George Lucas Educational Foundation. Disponible en: https://edut.to/2yfZA3c

Boullosa, P. (2017). Aprendizaje a fondo. En A. Grimaldo, G. Judson, P. Boullosa y S. Acuña, Educación imaginativa. Una aproximación a K. Egan (pp. 99-113). Madrid: Morata.

Bradley-Levine, J., Berghoff, B., Seybold, J., Sever, R. \& Blackwell, S. (2010). What teachers and administrators "need to know" about project-based learning implementation. Denver: Annual Meeting of the American Educational Research Association.

Brown, T. (2010). IDEO «design thinking» Approach. Disponible en: http://bit.ly/2tR1G7X

Buxarrais, $M^{a}$ R. (2013). Nuevos valores para una nueva sociedad. Un cambio de paradigma en educación. EDETANLA, 43, 53-65.

Cañal, P., Pozuelos, F. J. y Travé, G. (2005). Proyecto Curricular "Investigando Nuestro Mundo 6-12". Sevilla: Diada Editora.

Carbonell, J. (2014). Las pedagogías innovadoras y las visiones de los contenidos. Cuadernos de Pedagogía, 447, 42-45.

Carbonell, J. (2015). Pedagogías del siglo XXI. Alternativas para la innovación educativa. Barcelona: Octaedro.

Cieza, J. A. (2010). El compromiso y la participación comunitaria en los centros escolares. Un nuevo espacio-tiempo de intervención socioeducativa. Revista Interuniversitaria de Pedagogía Social, 17, 123-136.

Coll, C. (2006). Lo básico en la educación básica. Reflexiones en torno a la revisión y actualización del currículum de la educación básica. Revista Electrónica de Investigación Educativa, 8(1), 1-17.

Decroly, O. (1932). La función de globalización y la enseñanza. Madrid: Revista de Pedagogía.

Delval J. (2010). Hacia una escuela ciudadana. Madrid: Morata.

Dewey. J. (1918). Las escuelas de mañana. Madrid: Librería de los Sucesores de Hernando.

Dowden, T. (2007). Relevant, Challenging, Integrative and Exploratory Curriculum Design: Perspectives from Theory and Practice for Middle Level Schooling in Australia. Australian Educational Researcher, 34(2), 51-71.

Drake, S. M \& Burns, R. (2004). Integrated curriculum. Meeting, standards through. Virginia (USA): Association for Supervisión and curriculum Development.

Egan, K. (1991). La comprensión de la realidad en la educación infantily primaria. Madrid: Morata-MEC.

Egan, K. (1994). Fantasía e imaginación: su poder en la enseñanza. Madrid: Morata-MEC.

Egan. K. y Judson, G. (2018). Educación imaginativa. Herramientas cognitivas para el aula. Madrid: Narcea. Engel, S. (2011). Children's need to know: curiosity in schools. Harvard Educational Review, 81(4), 625645.

Fogarty, R. (2009). How to integrate the curricula. Thousand Oaks, CA: Corwin Press.

Franklin, B. M. y Johnson, C. C. (2006). Lo que enseñan las escuelas: una historia social del curriculum en los Estados Unidos desde 1950. Profesorado. Revista de Curriculum y Formación del Profesorado, 10(2), 1-29.

Fraser, D. (2000). Curriculum integration: What it is and is not. SET: Research Information for Teachers, 
3, 34-37.

Fraser, D. (2013). Curriculum integration. In B. Whyte, D. Fraser \& V. Aitkin (Eds.), Connecting curriculum, linking learning (pp. 18-33). Wellington: NZCER.

Freinet, C. (1927). L'imprimerie à l'école. Boulogne: Ferrary.

García Rubio, J. y Ros, A. (2018). El currículum básico como referente del derecho a la educación de calidad. EDETANIA, 53, 153-167.

García-Ajofrín, L. (2015). Finlandia sorprende con un nuevo currículum con menos contenidos y más interacción. Escuela. Disponible en: http://www.periodicoescuela.es.

García-Varcálcel, A. y Basilotta, V. (2017). Aprendizaje basado en proyectos: evaluación desde la perspectiva de alumnos de Educación Primaria. Revista de Investigación Educativa, 35(1), 113131.

Gil, D. (1987). Los errores conceptuales como origen de un nuevo modelo didáctico: de la búsqueda a la investigación. Investigación en la Escuela, 1, 36-41.

McGrath, D. (2002). Teaching on the Front Lines: using de Internet and Problem-Based Learning to enhance classroom teaching. Holist Nurs Pract, 16(2), 135-149.

Grimaldo, A., Judson, G., Boullosa, P. y Acuña, S. (2017). Educación imaginativa. Una aproximación a K. Egan. Madrid: Morata.

Guarro, A. (2001). Currículum democrático e integración curricular. Cooperación Educativa. Kikirikí, 59/60, 57-63.

Hargreaves, A., Earl, L., Moore, S. y Manning, S. (2001). Aprender a cambiar: la enseñanza más allá de las materias y los niveles. Barcelona: Octaedro.

Hernández, F. y Ventura, M. (1992). La organización del currículum por proyectos de trabajo. El conocimiento es un caleidoscopio. Barcelona: Graó.

Hoachlander, G. (1999). Integrating academic and vocational curriculum: Why is theory so hard to practice? Berkeley: National Center for Research in Vocational Education, University of California. Disponible en: http://bit.ly/38hTfS6

Howell, R. (2003). The importance of the project method in Technology Education. Journal of Industrial Teacher Education, 40(3), 1-7.

Imaginative Education Research Group -IERG- (2018). The learning in depth program. Disponible en: http://ierg.ca

Kilpatrick, W. H. (1918). The project method. Teachers College Record, 19, 319-335.

Lacueva, A. (2017). La investigación en la escuela-casa de la cultura. Caracas: Editorial Laboratorio Educativo.

Lam, C. C., Alviar-Martin, T., Adler, S. A. \& Sim, B. Y. (2013). Curriculum integration: Teachers' perspective and practice. Teaching and Teacher Education, 31, 23-34.

Lledó, Á. I. y Pozuelos, F. J. (1995) Ciencia y emotividad: el lugar de los planteamientos narrativos en la enseñanza elemental de las ciencias. Investigación en la Escuela, 25, 49-60.

Markham, T., Larmer, J. \& Ravitz, J. (2003). Project based learning bandbook: A guide to standards-focused project based learning for middle and high school teachers. Novato, CA: Buck Institute for Education.

Marsh, C.J. \& Willis, G. (2007). Curriculum: Alternative approaches, ongoing issues. Columbus: Pearson.

Martínez Celorrio, X. (2016). Innovación y reestructuración educativa en España: las escuelas del nuevo siglo. En A. Blanco y A. Chueca (Coord.), Informe España 2016. Madrid: Universidad Pontificia Comillas.

Meneses, N. (2020). La revolución “maker” llega a la educación. El País. Disponible en: https://bit.ly/2xk34Sv

Monereo, C. (2009). ¡Saquen los libros de texto! Resistencia, obstáculos y alternativas a la formación de los docentes para el cambio. Revista de Educación, 352, 583-597.

Mora, W. M. y García, Á. (1998). La resolución de problemas: una línea prioritaria de investigación 
en la enseñanza de las ciencias. Revista Educativa 'Volunt@d', 1, 14-19.

Pecore, J. L. (2015). From Kilpatrick's Project Method to Project-Based Learning. International Handbook of Progressive Education, 155-171.

Pérez Gómez, Á. I. (2012). Educarse en la era digital. Madrid: Morata.

Posner, G. J. (2004). Analizing the curriculum. Boston: McGraw Hill.

Pozo, J. I. (2016). Aprender en tiempos revueltos. La nueva ciencia del aprendizaje. Madrid: Alianza Ensayo.

Pozuelos, F. J. (2007). Trabajo por proyectos en el aula: descripción, investigación y experiencias. Morón (Sevilla): Cooperación Educativa. Ediciones MCEP.

Pozuelos, F. J. y Rodríguez, F. P. (2008). Trabajando por proyecto en el aula. Aportaciones de una investigación colaborativa. Investigación en la Escuela, 66, 5-27.

Pozuelos, F. J., Romero, D., García, F. J. y Morcillo, V. (2010). No basta con soñar otra escuela, hay que hacerla. Relato de experiencias y un caso. Investigación en la Escuela, 70, 5-20.

Sánchez Caballero, D. (2017). Asignaturas inabarcables dificultan a los alumnos asimilar los conocimientos. Eldiario.es. Disponible en: https://bit.ly/3aeHEUh

Savage, S. (2003). A guide to curricular integration. RedOrbit. Disponible en: https://bit.ly/34BaQ6t

Schumacher, D. H. (1995). Five Levels of Curriculum Integration Defined, Refined, and Described. Journal Research in Middle Level Education, 18, 73-94.

Silver, E.A., Smith, M. y Nelson, B. (1997). El proyecto QUASAR: los problemas de la equidad en la reforma de la enseñanza de las matemáticas en la educación secundaria. En W. G. Secada, E. Fennema y B. Adajian (Comps.), Equidad y enseñanza de las matemáticas: nuevas tendencias. (P Manzano. Trad.) (pp.23-70). Madrid: Morata-MEC.

Steinbeck, R. (2011). El "desing thinking" como estrategia de creatividad en la distancia. Comunicar, 19(37), 27-35. https:/ / doi.org/10.3916/C37-2011-02-02

Torres, J. (1994). Globalización e interdisciplinariedad: el curriculum integrado. Madrid: Morata.

Torres, J. (2014). Organización de los contenidos y relevancia cultural. Cuadernos de Pedagogía, 447, 5053.

Trujillo, F. (2019). ¿Cómo son los colegios más innovadores de España? The Conversation. Educación. Disponible en: http://bit.ly/2utERYt

Uruñuela, P. M. (2018). La metodología del Aprendizaje Servicio. Aprender a mejorar el mundo. Madrid: Narcea.

Vinyets, J. (s/f). Escuela, educación e innovación. ¿Design thinking? Disponible en: http://bit.ly/2ONH7QZ

Wert, C. (2019). Transformar esas antiguas aulas. En A. Moreno, E. Otero y M. Pozo, La nueva educación (pp. 546-571). Madrid: Residencia de Estudiantes. Fundación Giner de los Ríos y Acción Cultural Española.

Zorrilla, L., Capella, C. y Gil, J. (2016). Aprendizaje-servicio. En, Óscar Chiva y Manuel Martí (Coord.), Métodos pedagógicos y globalizadores (pp. 56-70). Barcelona: GRAÓ.

Zurdo, J. P. (2018). Con las manos en la placa: el movimiento "maker" llega a las aulas. El País. Revista Retina. Disponible en: https://bit.ly/34CDsw0 


\section{Información sobre los autores}

Autor: Francisco José Pozuelos Estrada

Institución: Área de Didáctica y Organización Escolar. Departamento de Pedagogía.

Email: pozuelos@,uhu.es

ORCID: https://orcid.org/0000-0002-1259-9917

Autor: Francisco Javier García Prieto

Institución: Área de Didáctica y Organización Escolar. Departamento de Pedagogía.

Email: fjavier.garcia@,dedu.uhu.es

ORCID: https://orcid.org/0000-0002-7427-830X 


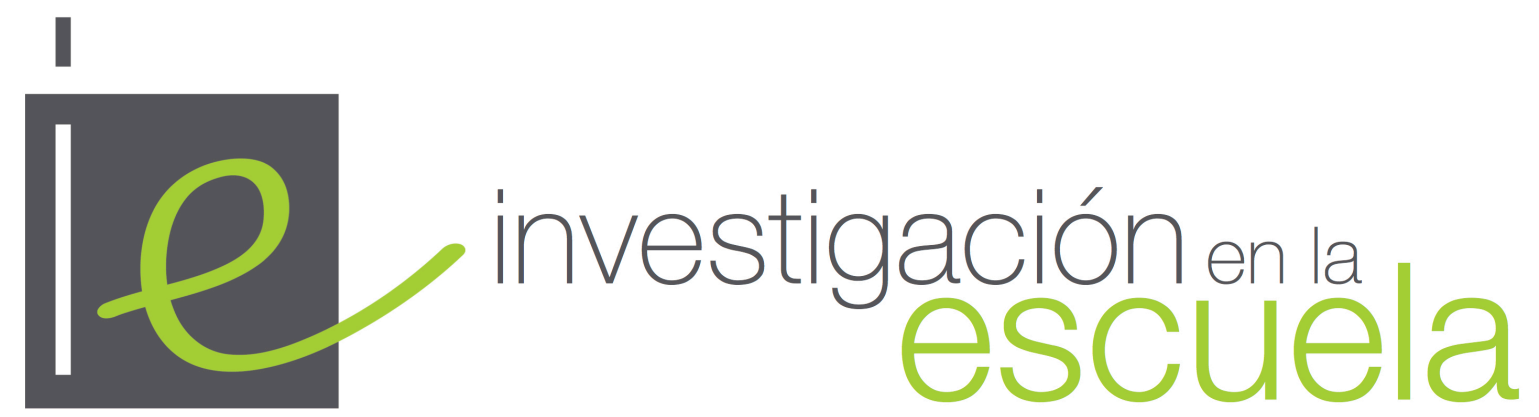

Revista académica evaluada por pares y de acceso abierto

Número 100

30 de abril de 2020

ISSN 2443-9991

\section{cc) (i) (2)}

Esta obra está bajo una licencia Creative Commons. Los/as lectores/as pueden compartir, copiar y redistribuir el material en cualquier medio o formato, así como adaptar, remezclar, transformar y construir a partir del material para cualquier propósito, incluso comercialmente. Para ello, deben de hacerlo bajo los siguientes términos: dando crédito de forma adecuada, brindando un enlace a la licencia e indicando si se han realizado cambios. Si se remezcla, transforma o crea a partir del material, debe distribuir su contribución bajo la misma licencia del original.

Más detalles de la licencia de CreativeCommons se encuentran en https://creativecommons.org/licenses/by-sa/4.0/deed.es

Cualquier otro uso debe ser aprobado en conjunto por el autor/es, o Investigación en la Escuela.

ư

Revista Editada por la Universidad de Sevilla. https://editorial.us.es/es/revistainvestigacion-en-la-escuela

Por errores y sugerencias contacte a investigacionenlaescuela@,us.es

La revista Investigación en la Escuela desde su origen en 1987 hasta su no 87 (2015) fue editada por Díada Editora. 
